\title{
The Provision of Digital Information in the Seat Comfort of the Seat Design
}

\author{
Kuen-Meau Chen ${ }^{1, *}$, Siu-Tsen Shen ${ }^{2}$, and Stephen D. Prior ${ }^{3}$ \\ ${ }^{1}$ Department of Industrial Design, National United University, \\ 1 Lien Da, Kung-Ching Li, Maioli 36003, Taiwan \\ kmchen0721@gmail.com \\ ${ }^{2}$ Department of Multimedia Design, National Formosa University, \\ 64 Wen-Hua Rd, Hu-Wei 63208, Taiwan \\ ${ }^{3}$ Department of Product Design and Engineering, Middlesex University, \\ London N14 4YZ, United Kingdom
}

\begin{abstract}
A great number of factors affect the uncomfortable of seats. Apart from external appearance, the most important is the comfort for users in designing an un-adjustable seat. A system or suggested principles which assist designers in designing the comfort of the seats to testees will benefit waist of users, and hence prevent them from potential discomfort and injuries. This study attempts to develop digital design platforms, and allows designers to measure the design scale of the comfort. We expect to find the association between seat parameter interaction and comfort. For example, when the seat height is much lower than the knee of testees, they can stretch calf forward if the space allows, so that their thighs contact the surface of the seat to share sciatic pressure in greater comfort. We believe to have changes of comfort in opposition to different seat parameter combinations. The final results will help effective reference parameters of comfort in seat design.
\end{abstract}

Keywords: Seat design, prediction model, dynamic interaction, Digital Design.

\section{Introduction}

While many factors influence the degree of seat comfort, few simple and practical tools are available for designers to assess seat design. The most important consideration when designing a non-adjustable chair is the comfort experienced by the targeted group. If these recommended guidelines are developed to help designers examine comfort in seat design, many users will benefit from better lumbar support by avoiding possible discomfort and injuries.

Many researchers have studied seat design and size recommendations, but there is little information about the interaction of various parameters related to seat size in the literature. For example, a seat with an angle close to 90 degrees between its back and surface usually causes strong discomfort to users; however, the degree of comfort can be improved if lumbar support is added. In addition, a seat that tilts back more than 10

\footnotetext{
${ }^{*}$ Corresponding author.
} 
degrees is worse for sitting in front of a low writing desk because it is difficult for users to lift their upper body when writing. This research should find other similar dynamic interactions to provide designers with simple guidelines to avoid reduced comfort when focusing on appearance. In addition, this study hope to understand the extremes of uncomfortable chairs, seat parameters' proportional effects on the degree of comfort, make an integrated prediction about comfort assessment, and further develop human factor design standards for the interaction of seat parameters.

\section{Literature Review}

People now spend long hours in a seat for work and leisure that results in musculoskeletal pain and discomfort [1]. However, the fit of seat size to body size does not necessarily determine comfort. Other factors include individual habits, seat pressure, types of tasks conducted when sitting, the muscles of different body parts, BMI, body fat percentage, allocation of body fat, thermal comfort resulting from the texture of the seat surface, and even microclimatic effects between the seat surface and the individual $[2,3,4]$. Many scholars have proposed various research perspectives and contributed valuable data for designers [5]. Eklund and Corlett (1987) compared two different types of seats and five different tasks. They found that seats or tasks that require individuals to bend the body-trunk often lead to more discomfort [6]. Zhao and Tang (1994) pointed out that better matching seat backs to users' backs and lumbar outlines significantly improves comfort [7]. Relevant studies suggest that comfort and discomfort are two independent indicators for evaluation [8]. Many studies only include subjective measurements of discomfort [9]. Some studies treat comfort and discomfort as opposite directions in one dimension [10]. De Looze et al. (2003) noticed that most researchers do not separate the comfort and discomfort but combine them when including comfort and discomfort as evaluation indicators [11]. Therefore, this research refers to the results of Kyunga and Nussbauma in 2008 and of Gyouhyung et al. in 2008. This study believes that measuring the concept of comfort, one can identify differences in comfort more effectively than differences in discomfort $[12,13]$.

This research focused on the interaction of seat parameters and proposed a prediction model for degrees of comfort. Why is the interaction between parameters so important? Relevant studies point out that the contents of tasks rather than the types of seats influence muscular tension [14]. This indicates that in addition to seat size, guidelines must consider the type of task. When carrying out different VDT tasks, the different heights of screens may cause physical discomfort [15]. Accordingly, seat comfort not only emphasizes physical size, but also involves the interaction with task environment and content. Consequently, to achieve a more comprehensive prediction, researchers should adopt a biomechanical model for calculating the lumbar pressure caused by certain postures and assess the surface pressure of seats [16].

\section{Research Method}

This experiment used adjustable chairs and asked participants to evaluate the degree of comfort of 27 differently sized seats based on three parameters: seat height, 
inclination angle of seat back, and height of lumbar support. By doing this, this study intended to discover the most comfortable seat and the interaction between parameters.

\subsection{Preparation before the Experiment}

Before the experiment, participants were asked to stand for 10 minutes to relieve the impact of sitting for a long period. This study measured participant knee height and the distance from the knees to the back of the buttocks. Participants were asked to take off shoes and remove belts and large items from pockets. Participants were asked to try out three different combinations of the parameters to understand the range of seat sizes and to prevent biased feelings during the experiment. Participants were told to evaluate different combinations of seat parameters based on preferred posture. Finally, terminology and evaluation standards were explained to the participants. The first phase of the experiment involved three parameters discussed the most in the literature.The selected sizes for the experiment were:

Factor 1 includes seat height at $33 \mathrm{~cm}, 39 \mathrm{~cm}$, and $45 \mathrm{~cm}$.

Factor 2 includes lumbar support as $15 \mathrm{~cm}, 27 \mathrm{~cm}$, and no lumbar support.

Factor 3 includes inclination angle of seat back as 90 degrees, 105 degrees, and 120 degrees.

\subsection{Digital Information of Seat Design}

The development of this system in accordance with human-scale planning, including gender, height, table height adjustment, seat and so the measurement of the size (see Fig. 1).

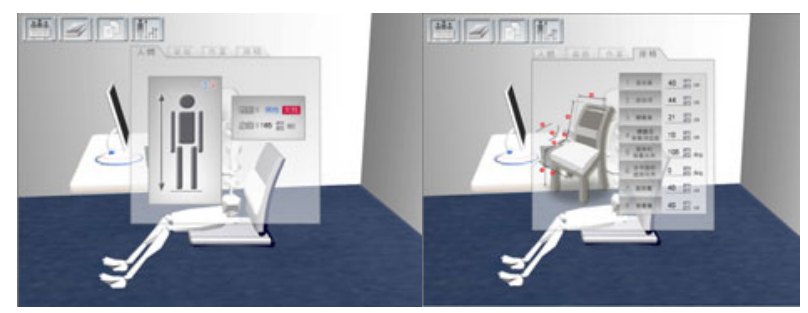

Fig. 1. Digital information system of seat design

\section{Discussion of the Results}

As presented in Table 1, this multivariate factor analysis reveals that the three major factors of seat height $(\mathrm{F}(2,944)=4.404, \mathrm{P}<0.05)$, height of lumbar support $(\mathrm{F}(2,944)=12.130, \mathrm{P}<0.05)$, and inclination angle of seat back $(\mathrm{F}(2,944)=47.297, \mathrm{P}<0.05)$ all have significant impact on comfort, and that there is significant interaction between the inclination angle of seat back and the height of lumbar support $(\mathrm{F}(4,944)=10.784, \mathrm{P}<0.05)$. 
Table 1. Three-Way ANOVA Analysis

\begin{tabular}{|c|c|c|c|c|c|}
\hline Variables & $\mathbf{S}$ & DOF & MS & $\mathbf{F}$ & $\mathbf{P}$ \\
\hline Seat height & 11.7 & 2 & 5.8 & 4.4 & $0.012 *$ \\
\hline Height of lumbar support & 32.4 & 2 & 16.2 & 12.1 & $0.000 *$ \\
\hline Inclination angle of the seat back & 126.5 & 2 & 63.2 & 47.2 & $0.000^{*}$ \\
\hline Seat height $*$ height of lumbar support & 8.6 & 4 & 2.17 & 1.6 & 0.166 \\
\hline Inclination angle of the seat back $*$ seat height & 14.7 & 4 & 3.6 & 2.7 & $0.027 *$ \\
\hline $\begin{array}{l}\text { Height of lumbar support* } \\
\text { inclination angle of seat back }\end{array}$ & 57.7 & 4 & 14.4 & 10.7 & $0.000^{*}$ \\
\hline $\begin{array}{l}\text { Seat height } * \text { Height of lumbar support* } \\
\text { Inclination angle of the back }\end{array}$ & 5.4 & 8 & 6 & .5 & 0.848 \\
\hline Error & 1228 & 918 & 1.3 & & \\
\hline Sum & 1485.7 & 944 & & & \\
\hline
\end{tabular}

Table 1 reveals the significant interaction between seat height and the inclination angle of the seat back $(\mathrm{F}(4,944)=2.764, \mathrm{P}<0.05)$. Making the inclination angle larger than 105 degrees significantly improves comfort. Another important finding is a higher $(45 \mathrm{~cm})$ seat causes less discomfort than a seat with a lower $(33 \mathrm{~cm})$ surface, even if the inclination angle is 90 degrees. A higher seat causes the least discomfort compared to seats with different heights so long as the inclination angle is larger (120 degrees). No significant difference was found between seat height and the height of lumbar support in the multivariate factor analysis $(\mathrm{F}(4,944)=1.624, \mathrm{P}>0.05)$.

\section{Conclusion}

The three factors adopted in this experiment are the most common and important parameters when designing seats. Seats with an inclination angle of 90 degrees but have no lumbar support receive low comfort scores. The discomfort may result from having to excessively bend the body trunk causing pressure on the abdominal cavity and the lumbar vertebra cannot retain a concave posture. By contrast, when the inclination angle is 120 degrees, seats without lumbar support are more comfortable. A possible explanation is that when the upper body remains straight, the lumbar outline forms a deeper concave; however, when the angle of the back is 120 degrees, the outline of the back is changed, and the concave of the lumbar vertebra is no longer obvious. Lumbar support of the same thickness may cause pressure to some tissues and lead to discomfort. From the perspective of lumbar support and seat back inclination angle, this study makes two observations. First, when the inclination angle is close to 90 degrees, increasing lumbar support can improve comfort. Second, when the inclination angle is larger than 120 degrees, removing lumbar support or reducing the thickness of the lumbar cushion relieves pressure on the lumbar area.

Acknowledgement. This study is supported by National Science Counsel, Taiwan, with project number NSC-98-2511-S-239-001-MY2.

\section{References}

1. Ebe, K., Griffin, M.J.: Factors affecting static seat cushion comfort. Ergonomics 44(10), 901-921 (2001)

2. Gyi, D.E., Porter, J.M.: Interface pressure and the prediction of car seat discomfort. Applied Ergonomics 30, 99-107 (1999) 
3. Nick, C., Brynley, P., Hull, G.E.: An empirical study of preferred settings for lumbar support on adjustable office chairs. Ergonomics 41(4), 401-409 (1998)

4. Diebschlag, W., Heidinger, F., Kuurz, B., Heiberger, R.: Recommendation for ergonomic and physiological climatic vehicle seat design. Society of Automotive Engineers, T Paper no. 880055 (1988)

5. Zhang, L., Helander, M., Drury, C.: Identifying factors of comfort and discomfort. Human Factors 38(3), 377-38 (1996)

6. Eklund, J.A.E., Corlett, E.N.: Evaluation of spinal loads and chair design in seated work tasks. Clinical Biomechanics 2, 27-33 (1987)

7. Zhao, J.H., Tang, L.: An evaluation of comfort of a bus seat. Applied Ergonomics 25, 386392 (1994)

8. Sauter, S.L., Swanson, N.G., Waters, T.R., Hales, T.R., Dunkin-Chadwick, R.: Musculoskeletal discomfort surveys used at NIOSH. In: Stanton, N., Hedge, A., Brookhuis, K., Salas, E., Hendrick, H. (eds.) Handbook of Human Factors and Ergonomics Methods. CRC Press, Boca Raton (2005)

9. Hsu, W.-H., Wang, M.-J.: Physical discomfort among visual display terminal users in a semiconductor manufacturing company: a study of prevalence and relation to psychosocial and physical/ergonomic factors. American Industrial Hygiene Association Journal 64(2), 276-282 (2003)

10. Kee, D., Karwowski, W.: Joint angles of isocomfort for female subjects based on the psychophysical scaling of static standing postures. Ergonomics 47(4), 427-445 (2004)

11. De Looze, M.P., Kuijt-Evers, L.F.M., van Dieen, J.: Sitting comfort and discomfort and the relationships with objective measures. Ergonomics 46(10), 985-997 (2003)

12. Gyouhyung, K., Maury, A., Nussbaum, Kari, B.R.: Driver sitting comfort and discomfort (part I): Use of subjective ratings in discriminating car seats and correspondence among ratings. International Journal of Industrial Ergonomics 38, 516-525 (2008)

13. Kyunga, G., Nussbauma, M.A., Babski-Reevesb, K.: Driver sitting comfort and discomfort (part I): Use of subjective ratings in discriminating car seats and correspondence among ratings. International Journal of Industrial Ergonomics 38, 516-525 (2008)

14. Van Dieen, J.H., De Looze, M.P., Hermans, V.: Effects of dynamic chairs on trunk kinematics, trunk extensor EMG and spinal shrinkage. Ergonomics (2001) ISSN: 00140139

15. Seghers, J., Jochen, A., Spaepen, S.: Posture, muscle activity and muscle fatigue in prolonged VDT work at different screen height settings. Ergonomics 46(7), 714-730 (2003)

16. Kayis, B., Hoang, K.: Static three-dimensional modeling of prolonged seated posture. Applied Ergonomics 30(3) (1999) 